\title{
Widefield Optical and AFM Analysis of Few Layer Graphene Nanowires Functionalized with DNA
}

\author{
Masudur Rahman and Michael L. Norton \\ Department of Chemistry, Marshall University, One John Marshall Drive, Huntington, WV 25755
}

Progress in molecular sensor development requires the production of nanostructures which are intimate connection to, and present in a controllable manner, molecular species which can interact specifically with an analyte. Highly oriented pyrolytic graphite (HOPG) is still one of the best sources for highly crystalline graphene layers. Graphene, which constitutes a single layer of graphite, displays many of the properties of value in a nanosensor material. Particularly it has very high intrinsic electron mobility [1], and excellent mechanical properties [2]. Here we will present in detail methods employed for microscopic characterization of HOPG patterned at both the micro and 100 nanometer scale and subsequently functionalized with short DNA capture strands. Large scale patterns were produced lithographically using a 5micron bar TEM grid as a plasma resist. Finer patterns consisting of long $200 \mathrm{~nm}$ wide multilayer grapheme lines were prepared on HOPG substrates using interference lithography to expose resist and oxygen plasma etching. These approaches and the resulting structures are presented in Figure 1. Figure 2 presents a schematic of the process for preparing samples of Rhodamine labeled DNA sequences conjugated to the 5 micron square bar pattern and to the $200 \mathrm{~nm}$ graphene line pattern.

The patterned HOPG, at the perimeter of the pattern, is presumed to be oxidized carbon, containing terminal carboxylate groups. It has been demonstrated that such terminal carboxylate groups can be activated for the formation of a stable amide bonds with biomolecules [3]. Using this functionalization method, single stranded DNA sequences have been covalently immobilized to the edges of these structures. Next, complementary DNA sequences fluorescently labeled with Rhodamine were annealed with the constructs to enable hybridization. The complete DNA/graphene/graphite assemblies were printed onto the surface of PDMS substrates using soft lithography. The progresses of the lithographic processes were followed using AFM. Due to the nature of the DNA constructs, the DNA process was monitored using wide field fluorescence optical microscopy.

All fluorescence images were taken with a Rolera-MGi camera (QIMAGING), with acquisition parameters: gain 2, EM gain 3500, exp. Time $=100 \mathrm{msec}$, display range 400-8000 counts. Two color fluorescence imaging was performed using an Optosplit (CAIRN, ENGLAND) system with filter characteristics for the green channel (left) of excitation 470/30 and emission 515/30, and for the red channel (right) excitation 550/20 and emission 595/50. Optosplit microscopy images showed that Rhodamine-DNA sequences bind at the perimeter of the large scale patterns and that these patterns can be printed consistently onto PDMS. DNA binding occurs at high density along the edges. The binding density can be monitored via wide field fluorescence microscopy and may be controlled by changing sample preparation conditions. 


\section{References:}

[1] C. Berger, et al., "Electronic confinement and coherence in patterned epitaxial graphene," Science, vol. 312, pp. 1191-6, May 262006.

[2] A. Sidorov, et al., "Electrostatic deposition of graphene in a gaseous environment: a deterministic route for synthesizing rolled graphenes?," Nanotechnology, vol. 20, p. 055611, Feb 42009.

[3] X. Guo, et al., "Conductivity of a single DNA duplex bridging a carbon nanotube gap," Nat Nanotechnol, vol. 3, pp. 163-7, Mar 2008.

\section{Figures:}
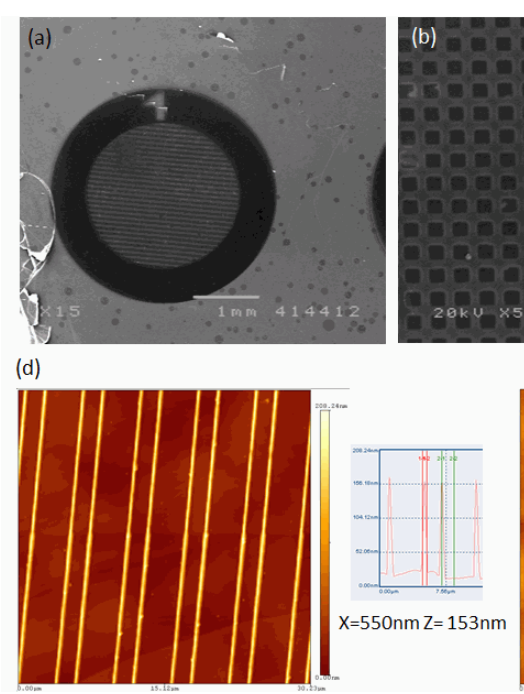
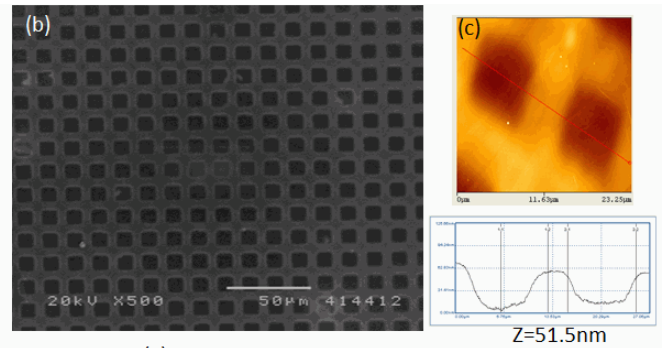

(e)
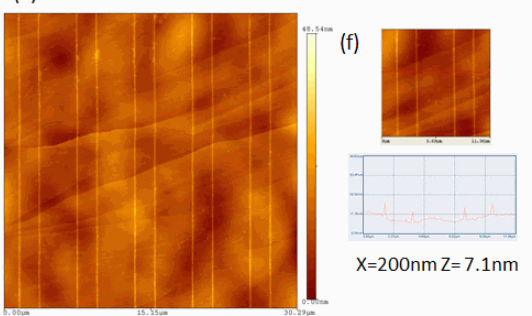

Figure 1: a) SEM images of HOPG surface after $\mathrm{O}_{2}$ plasma etching, round shape of TEM grid replica is clearly visible; (b) higher resolution SEM image of pattern etched into HOPG; (c) AFM analysis of TEM grid pattern, demonstrating $Z=51.5 \mathrm{~nm} / 40 \mathrm{~min}$ etching rate; (d) AFM image of resist pattern of interference lithography on HOPG (e,f) AFM image of graphene line pattern on HOPG, demonstrating $\mathrm{Z}=7.1 \mathrm{~nm}$.

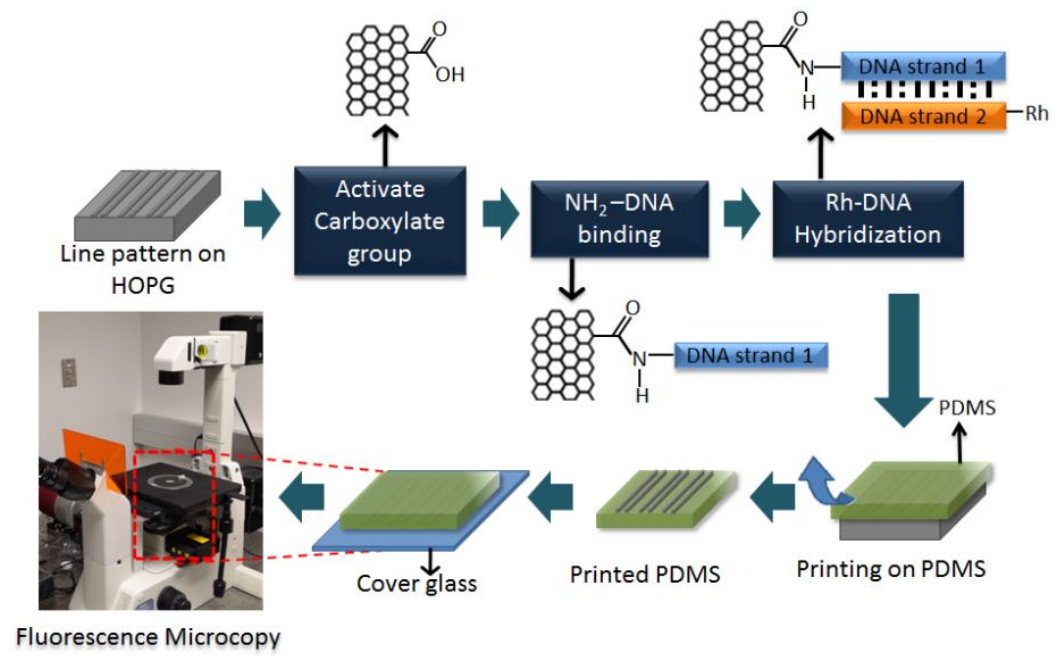

Figure 2: (a) Schematic illustration shows the printing method of the Rh-labeled patterned graphene onto PDMS. 